\title{
Anti-PD-1 Therapy plus Chemotherapy and/or Bevacizumab as Second Line or later Treatment for Patients with Advanced Non-Small Cell Lung Cancer
}

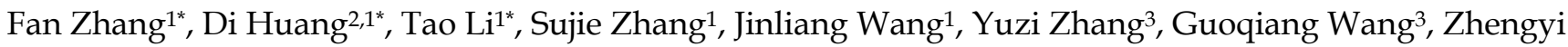
Zhao $^{3}$, Junxun Ma${ }^{1}$, Lijie Wang ${ }^{1}$, Danyang Sun ${ }^{1}$, Pengfei Cui ${ }^{1}$, Shangli Cai ${ }^{3}$, Shunchang Jiao ${ }^{1}$, Lei Zhao ${ }^{4}{ }^{\bowtie},{ }^{1}$ $\mathrm{Hu}^{1,2}{ }^{\mathbb{}}$

1. Department of Oncology, Chinese PLA General Hospital, PLA School of Medicine, Beijing, People's Republic of China

2. School of medicine, Nankai University, Tianjin, People's Republic of China

3. The Medical Department, 3D Medicines Inc., Shanghai, People's Republic of China

4. National Clinical Research Center for Normal Aging and Geriatric \& The Key Lab of Normal Aging and Geriatric, Institute of Geriatric, PLA General

Hospital, Beijing, People's Republic of China

*Three authors contributed equally to this work.

$\triangle$ Corresponding authors: Yi Hu, Department of Oncology, Chinese PLA General Hospital, 28 Fuxing Road, Haidian, Beijing 100853, P.R. China. School of medicine, Nankai University, Tianjin, People's Republic of China. Tel: +86-13911031186, Fax: +86- 010-66939272, Email: huyi0401@aliyun.com. Lei Zhao, National Clinical Research Center for Normal Aging and Geriatric \& The Key Lab of Normal Aging and Geriatric, Institute of Geriatric, Chinese PLA General Hospital, 28 Fuxing Road, Haidian, Beijing 100853, P.R. China. Tel: +86-15210661966, E-mail: jackyzhao010@126.com.

(C) The author(s). This is an open access article distributed under the terms of the Creative Commons Attribution License (https://creativecommons.org/licenses/by/4.0/). See http://ivyspring.com/terms for full terms and conditions.

Received: 2019.06.27; Accepted: 2019.09.15; Published: 2020.01.01

\begin{abstract}
Immune checkpoint inhibitor combination therapy exhibited outstanding efficacy in first line setting for advanced non-small cell lung cancer (aNSCLC) patients. However, whether PD-1 inhibitor combined treatment is effective in second line or later setting remains unknown. Therefore, we retrospectively evaluated the efficacy of combined therapy of PD-1 inhibitor with chemotherapy and/or bevacizumab compared to PD-1 inhibitor alone for aNSCLC patients in second line or later setting. Patients with aNSCLC who have received anti-PD-1 based therapy between 2015 and 2017 were screened, and 55 patients were ultimately included and divided into the monotherapy group $(\mathrm{N}=33)$ and the combination group $(\mathrm{N}=22)$. Patients treated with combination therapy exhibited superior PFS versus those treated with monotherapy (median PFS, 7.5 months vs 3.3 months; hazard ratio $0.28 ; 95 \% \mathrm{Cl}, 0.14-0.56 ; P<0.001)$. Objective response rate and disease control rate were $31.8 \%(7 / 22)$ and $95.5 \%(21 / 22)$ in the combination group and $10.0 \%(3 / 30)$ and $46.7 \%(14 / 30)$ in the monotherapy group, respectively (ORR, $P=0.075$; $D C R, P<0.001$ ). Five patients $(22.7 \%)$ experienced grade $3-4$ adverse events in the combination group and two patients $(6.1 \%)$ in the monotherapy group. Taken together, our results indicated that for NSCLC patients who had failed on the first-line or later treatment, PD-1 inhibitor in combination with chemotherapy and/or bevacizumab might be a favorable treatment option. These findings warrant further validation in prospective studies.
\end{abstract}

Key words: immune checkpoint inhibitor, combination therapy, non-small cell lung cancer

\section{Introduction}

Non-small cell lung cancer (NSCLC) is currently the most common cause of cancer death worldwide.1,2 Immune checkpoints inhibitors (ICIs) such as monoclonal antibodies against programmed cell death protein-1 (PD-1 inhibitor) or its ligand (PD-L1 inhibitor) have revolutionized the clinical management of patients with aNSCLC. ${ }^{3-6}$ In patients with previously treated aNSCLC, ICIs exhibited a 
substantial improvement of prognosis compared to standard chemotherapy, as demonstrated by an improvement of five-year overall survival (OS) rate from $4.9 \%$ to $16 \% .^{7}$

However, effective treatment strategies are still limited for aNSCLC patients who failed after the first-line treatment. Even though FDA has approved ICIs (pembrolizumab, nivolumab, atezolizumab) in advanced NSCLC for second-line therapy, only a portion of unselected aNSCLC patients would benefit from ICIs monotherapy, with an objective response rate (ORR) ranging from $14 \%$ to $20 \% .4,5,8,9$ Multiple predictive biomarkers provide assistance to distinguish the patients sensitive to ICIs, such as expression of PD-L1 protein, MSI-H/dMMR and tumor mutational burden, but a large amount of patients do not harbor these biomarkers and maybe less likely to respond to ICIs monotherapy.3,10,11 Moreover, patients with NSCLC harboring driver mutations (especially EGFR/ALK) tended to develop resistance to TKI treatment eventually. Of these, the response rate of single-agent ICIs appears to be less reported. ${ }^{12-14}$ In this case, an appropriate method to improve the efficacy of ICIs as second-line or later treatment was required.

Current efforts are focusing on developing new rational ICIs combination strategies to augment the ORR of ICIs. Increasing evidences indicated that additional treatments up-regulating the release and presentation of tumor-specific neoantigen would synergize with ICIs, such as chemotherapy and radiotherapy. ${ }^{15,16}$ In addition, angiogenesis inhibitors could also synergistically act with ICIs by modulating tumor microenvironment and promoting immune cell infiltration. ${ }^{17,18}$ As such, a number of clinical trials were conducted to investigate whether ICIs combination therapy is effective as first-line treatment in aNSCLC. For example, pembrolizumab in combination with carboplatin-containing chemotherapy have exhibited superior ORR (55\% vs. 29\%), PFS (13.0 months vs. 8.9 months), and OS (1-year OS rate: $69.2 \%$ vs. $49.4 \%$ ) for aNSCLC patients in treatment-naïve setting as demonstrated from the phase II KEYNOTE-021 trial ${ }^{19}$ and the phase III KEYNOTE-189 trial20, respectively. KEYNOTE-407, another double-blind phase III trial in metastatic squamous NSCLC, also showed that pembrolizumab plus chemotherapy (carboplatin/(nab-)paclitaxel) could provide significant benefit versus chemotherapy regarding PFS and OS.21 Phase I clinical trial CHECKMATE-012 suggested an encouraging activity of nivolumab in combination with paclitaxel-carboplatin chemotherapy, with an ORR of $47 \%$ and a 2 -y OS rate of $62 \% .{ }^{22}$ Furthermore, the phase III clinical trial IMpower150 showed that first-line therapy of atezolizumab plus bevacizumab and chemotherapy (paclitaxel-carboplatin) reduced $38 \%$ risk of death $(\mathrm{HR}=0.62)$ compared with bevacizumab and chemotherapy for non-squamous NSCLC patients without EGFR/ALK mutation. ${ }^{23}$ Similarly, results from IMpower131 also demonstrated a greater benefit regarding PFS in advanced squamous NSCLC patients receiving atezolizumab combination therapy compared with chemotherapy alone. ${ }^{24}$ Taken together, these data indicated that ICIs combination strategies may offer promising opportunities for advanced NSCLC patients in treatment-naïve setting.

However, by far, limited data is available for the combination therapy of ICIs for aNSCLC patients in second-line or later settings. Therefore, we carried out this analysis to retrospectively explore the efficacy of PD-1 inhibitor combination therapy as a second-line or later treatment compared with anti-PD-1 monotherapy in aNSCLC.

\section{Materials and Methods}

\section{Study Design and participants}

Advanced NSCLC patients who have received PD-1 inhibitor based monotherapy or combination therapy at General Hospital of the People's Liberation Army (GHPLA) between March 2015 and July 2017 were screened. A total of 92 patients were identified (Figure 1). Patients who failed the first-line treatment were eligible for this analysis. Moreover, patients were excluded, if: (1) combined drugs beyond chemotherapy (chemo) and bevacizumab (beva); and (2) combined with radiotherapy. Treatment strategies were made by the physicians based on tumor molecular profiling, efficacy of previous line therapy, toxicity, patient's physical condition, and patient's decision. Due to the low response rate of PD-1 inhibitor monotherapy reported previously and the expensive costs of drugs, some patients would rather try the combination therapy which may exhibit a higher possibility of disease response. Drug dose and cycle were given according to the instructions. All patients provided written informed consent. Ethics Committee of GHPLA approved the study.

\section{Data Collection}

This was a retrospective study based on prospectively collected data. Based on the pre-designed CRF, two physicians independently extracted and verified the information from the medical records for the clinicopathologic and treatment features. Tumor imaging assessments for advanced NSCLC patients were done by the oncologist per RECIST (version 1.1) routinely every $6 \sim 8$ weeks. ${ }^{25}$ PFS was defined by the time interval 
from treatment initiation to tumor progression or death. Patients were censored on the date of last visit if no documented disease progression occurred. The imaging data of all the included patients were independently assessed by two radiologists. When the evaluation results were inconsistent, the results would be evaluated by the director of imaging center. The National Cancer Institute Common Terminology Criteria for Adverse Events, version 4.0, was used to evaluate the adverse events (AEs).

\section{Study Objectives}

We report the study according to Transparent Reporting of Evaluations with Nonrandomized Designs (TREND). ${ }^{26}$ The primary objective was PFS. The secondary objectives included objective response rate (ORR), disease control rate (DCR) and safety profile.

\section{Statistical Analysis}

All statistical analyses were conducted with GraphPad Prism software version 7.01 (GraphPad Software, Inc., USA) and SPSS statistical software version 20.0 (IBM Corp., USA). Mean \pm SD with the use of $\mathrm{T}$ testing was selected for continuous or ordinal variables with normally distributions; otherwise, median \pm SD with the use of Mann-Whitney U test was selected. Chi-squared test or Fisher's exact test was used to assess associations between categorical variables. All $P$ values were two-sided with $P<0.05$ to be considered as statistically significant.
Kaplan-Meier survival curves for PFS were generated and compared with a stratified log-rank test. Hazard ratios (HR) and associated 95\% CIs were provided by Cox's regression. Variables that achieved $\mathrm{P} \leq 0.05$ or might have an important effect on prognosis were entered into multivariable models. The missing data was not analyzed.

\section{Results}

\section{Cohort characteristics and treatment}

In total, 55 patients with aNSCLC were included in the studied cohort, and all of them have received PD-1 inhibitor for the second-line or later treatment. (Figure 1). Amongst all, there were 22 patients in the combination therapy group and 33 patients in the monotherapy group. All patients have progressed after systemic chemotherapy for metastatic disease. A total of $50(90.9 \%)$ patients in this study have failed after platinum-based chemotherapy previously. Combination treatments received by each individual is shown in Table S1 and $40.9 \%$ of the patients received nab-paclitaxel. In general, clinicopathologic features were balanced between the two groups (Table 1), with slight imbalances in the proportion of lung squamous cancer population and performance status KPS of 90 . About half of the patients were never smokers which was higher than seen in patients treated in clinical trials of PD-1. In addition, one third of the patients had developed metastasis of brain.

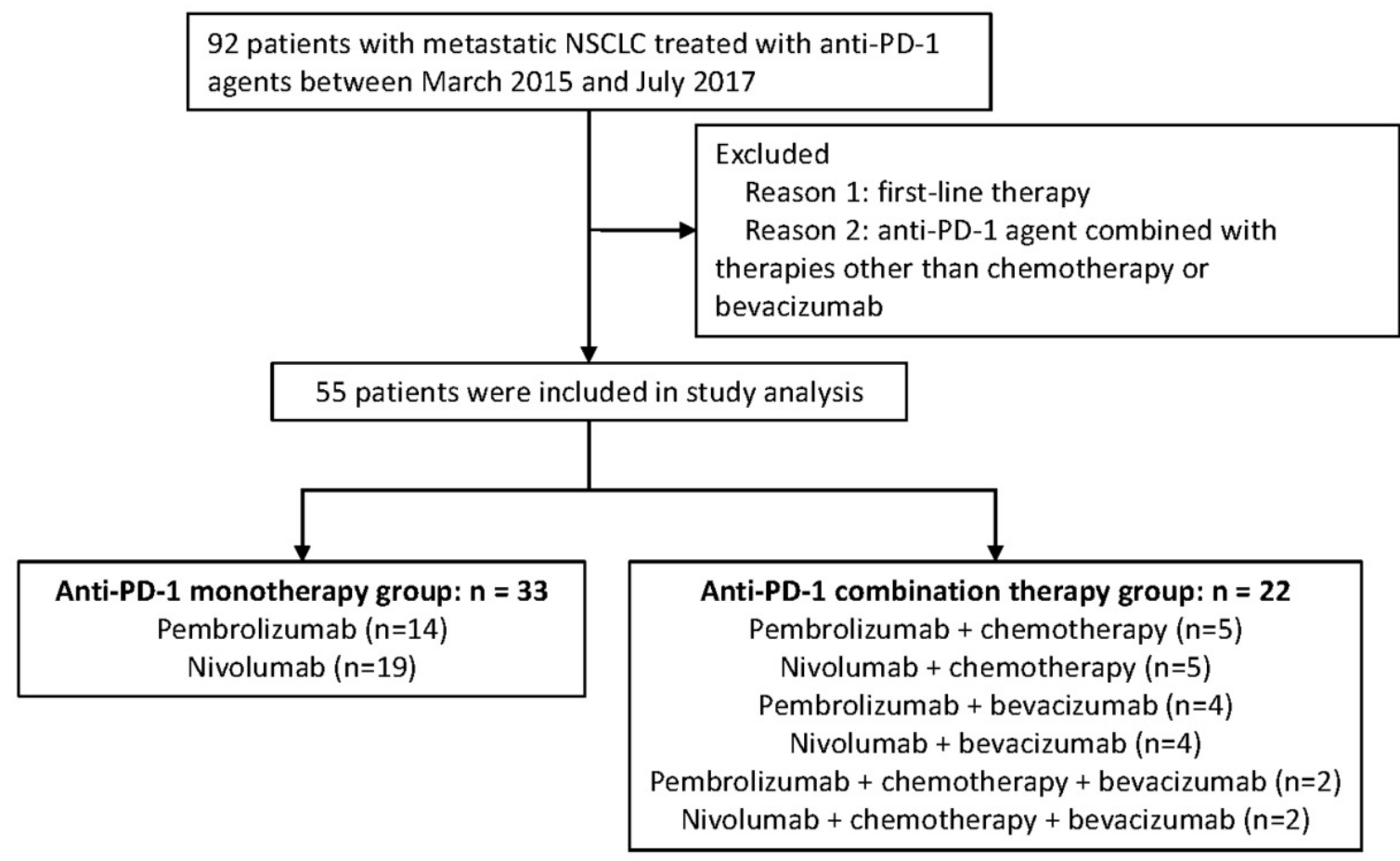

Figure 1. Diagram of the study. 
Table 1. Demographics and baseline characteristics.

\begin{tabular}{|c|c|c|c|}
\hline Characteristic & $\begin{array}{l}\text { Anti-PD-1 combination } \\
\text { therapy } \\
\mathrm{N}=22\end{array}$ & $\begin{array}{l}\text { Anti-PD-1 } \\
\text { monotherapy } \\
\mathrm{N}=33\end{array}$ & $P$ value \\
\hline Median age, years (range) & $54(33-79)$ & $56(38-80)$ & 0.214 \\
\hline Sex, n (\%) & & & 0.762 \\
\hline Male & $17(77.3 \%)$ & $24(72.7 \%)$ & \\
\hline Female & $5(22.7 \%)$ & $9(27.3 \%)$ & \\
\hline Tumor histology, n (\%) & & & 0.391 \\
\hline Squamous & $6(27.3 \%)$ & $14(42.4 \%)$ & \\
\hline Adenocarcinoma & $16(72.7 \%)$ & $19(57.6 \%)$ & \\
\hline Smoking history, $\mathbf{n}(\%)$ & & & 0.945 \\
\hline Former or Current & $11(50.0 \%)$ & $16(48.5 \%)$ & \\
\hline Never & $10(45.5 \%)$ & $15(45.5 \%)$ & \\
\hline Unknown & $1(4.5 \%)$ & $2(6.0 \%)$ & \\
\hline EGFR status, $\mathrm{n}(\%)$ & & & 0.467 \\
\hline Wild type & $7(31.8 \%)$ & $15(45.6 \%)$ & \\
\hline Mutant & $4(18.2 \%)$ & $3(9.0 \%)$ & \\
\hline Unknown & $11(50.0 \%)$ & $15(45.4 \%)$ & \\
\hline ALK translocation & & & 0.214 \\
\hline No & $7(31.8 \%)$ & $16(48.5 \%)$ & \\
\hline Yes & $1(4.5 \%)$ & 0 & \\
\hline Unknown & $14(63.7 \%)$ & $17(51.5 \%)$ & \\
\hline \multicolumn{2}{|c|}{ Performance status (KPS), n (\%) } & & 0.209 \\
\hline 90 & $19(86.4 \%)$ & $20(60.6 \%)$ & \\
\hline 80 & $1(4.5 \%)$ & $6(18.2 \%)$ & \\
\hline 70 & $2(9.1 \%)$ & $5(15.2 \%)$ & \\
\hline$<70$ & 0 & $2(6.0 \%)$ & \\
\hline \multicolumn{2}{|c|}{ Prior lines for metastatic disease } & & 0.941 \\
\hline 1 & $6(27.3 \%)$ & $10(30.3 \%)$ & \\
\hline 2 & $7(31.8 \%)$ & $11(33.3 \%)$ & \\
\hline$\geq 3$ & $9(40.9 \%)$ & $12(36.4 \%)$ & \\
\hline \multicolumn{4}{|l|}{ Metastatic site } \\
\hline Brain & $7(31.8 \%)$ & $10(30.3 \%)$ & 1.000 \\
\hline Liver & $3(13.6 \%)$ & $6(18.2 \%)$ & 0.727 \\
\hline Bone & $6(27.3 \%)$ & $10(30.3 \%)$ & 0.769 \\
\hline
\end{tabular}

Table 2. Univariable and Multivariable Analysis of Progressionfree Survival

\begin{tabular}{|c|c|c|c|c|c|c|}
\hline \multirow[b]{2}{*}{ Parameter } & \multicolumn{3}{|c|}{ Univariable Analysis } & \multicolumn{3}{|c|}{ Multivariable Analysis } \\
\hline & HR & $95 \% \mathrm{CI}$ & $P$ & HR & $95 \% \mathrm{CI}$ & $P$ \\
\hline \multicolumn{7}{|l|}{ Age } \\
\hline$<65 v \geq 65$ & 0.793 & $0.353-1.783$ & 0.575 & & & \\
\hline \multicolumn{7}{|l|}{ Sex } \\
\hline Male $v$ female & 1.167 & $0.601-2.266$ & 0.647 & & & \\
\hline \multicolumn{7}{|l|}{ Smoking status } \\
\hline Former/current $v$ never & 0.932 & $0.692-1.254$ & 0.641 & & & \\
\hline \multicolumn{7}{|l|}{ Performance status(KPS) } \\
\hline $90 v \leq 80$ & 0.427 & $0.228-0.798$ & 0.008 & 1.721 & $0.898-3.296$ & 0.102 \\
\hline \multicolumn{7}{|l|}{ Tumor histology } \\
\hline $\begin{array}{l}\text { Squamous } v \\
\text { adenocarcinoma }\end{array}$ & 0.851 & $0.458-1.584$ & 0.611 & & & \\
\hline \multicolumn{7}{|l|}{ LDH level at baseline } \\
\hline$<200 v \geq 200$ & 0.863 & $0.476-1.563$ & 0.626 & & & \\
\hline \multicolumn{7}{|l|}{ EGFR/ALK status } \\
\hline Mutant $v$ wild type & 0.735 & $0.293-1.844$ & 0.512 & & & \\
\hline \multicolumn{7}{|l|}{$\begin{array}{l}\text { Prior lines for metastatic } \\
\text { disease }\end{array}$} \\
\hline $1 v \geq 2$ & 1.365 & $0.732-2.547$ & 0.327 & & & \\
\hline \multicolumn{7}{|l|}{ Metastatic site } \\
\hline \multicolumn{7}{|l|}{ Brain } \\
\hline Yes $v$ no & 0.989 & $0.721-1.357$ & 0.945 & & & \\
\hline \multicolumn{7}{|l|}{ Liver } \\
\hline Yes $v$ no & 0.945 & $0.644-1.388$ & 0.774 & & & \\
\hline \multicolumn{7}{|l|}{ Bone } \\
\hline Yes $v$ no & 1.040 & $0.754-1.432$ & 0.812 & & & \\
\hline \multicolumn{7}{|l|}{ Anti-PD-1 agents } \\
\hline $\begin{array}{l}\text { Pembrolizumab } v \\
\text { nivolumab }\end{array}$ & 1.323 & $0.734-2.385$ & 0.353 & & & \\
\hline \multicolumn{7}{|l|}{ Treatment group } \\
\hline $\begin{array}{l}\text { Combination } v \\
\text { monotherapy }\end{array}$ & 0.282 & $0.143-0.555$ & $<0.000$ & 0.319 & $0.158-0.645$ & 0.001 \\
\hline
\end{tabular}

\section{Efficacy}

The median PFS was 7.5 months (95\% confidence interval $[\mathrm{CI}], 6.8-13.1)$ in the combination therapy group and 3.3 months $(95 \% \mathrm{CI}, 2.6-4.8)$ in the monotherapy group (hazard ratio [HR], $0.28 ; 95 \% \mathrm{CI}$, 0.14-0.56; $P<0.001$ ) (Figure 2). In univariable logistic regression analysis, performance status score at 90 was also associated with better PFS compared with less than 90 (Table 2). In multivariable cox models where age, sex, performance status score, prior lines for metastatic, smoking history, and treatment group were included, combination therapy group remained significant (HR, 0.32; 95\% CI 0.16-0.65; $P=0.001)$. The hazard ratios for PFS significantly favored combination therapy across most subgroups (Figure 3). The ORR was relatively higher in the combination therapy than that in the monotherapy group $(31.8 \%$ [95\% CI, 15.9-51.5] vs $10.0 \%$ [95\% CI, 2.8-23.8]; $P=$ 0.075) (Table S2). In the subgroup analysis of the combination therapy group, the objective response rate was $40 \%(4 / 10)$ in anti-PD-1 plus chemo, $0 \%$ $(0 / 8)$ in anti-PD-1 plus beva and $75 \%(3 / 4)$ in anti-PD-1 plus chemo/beva. The DCR was significantly higher for patients receiving combination therapy versus monotherapy (95.5\% [95\% CI 80.2-99.8] vs 46.7\% [95\% CI 33.8-63.1]; $P<$ $0.001)$. Overall, $9 / 30(30 \%)$ patients in monotherapy group and 14/22 (63.6\%) patients in combination therapy group had a tumor decrease from baseline in the target lesions (Figure 4). Median change was 5\% (IQR -10 to 30$)$ with monotherapy and $-7.5 \%$ (-35 to 5) with combination therapy (Figure 4).

\section{Adverse events}

AEs of any grade occurred in $95.5 \%(21 / 22)$ with combination therapy and $87.9 \%$ (28/33) with monotherapy. AEs are summarized in Table 3 . Consistent with reported observations, fatigue (7 [31.8\%]), nausea (6 [27.3\%]) and rash (4 [18.2\%]) were the most common AEs of any grade in the combination therapy group ${ }^{19,22}$. No death occurred. Grade 3 to 4 AEs were observed in 22.7\% (5/22) with combination therapy, which is relatively higher than that in the monotherapy group (2/33, [6.1\%]) although no significant statistical difference was detected $(P=0.10)$. The most common AEs included leucopenia $(2 / 22$ [9.1\%]), pneumonitis $(1 / 22$ [4.5\%]), and fever $(1 / 22[4.5 \%])$ in the combination group, and fever $(1 / 33[3.0 \%])$, rash $(1 / 33[3.0 \%])$ and nausea $(1 / 33[3.0 \%])$ in the monotherapy group, respectively.

\section{Discussions}

The results of this retrospective analysis displayed superiority of PD-1 inhibitor combination therapy over its monotherapy for aNSCLC patients in 
second-line or beyond setting. The median PFS in the monotherapy group was similar with previous studies. $4,5,8$ The combined therapy of PD-1 inhibitor plus chemo and/or beva was associated with superior PFS, elevated DCR and a tendency of higher ORR. The frequencies of adverse events in grade 3-4 were relatively higher with the combined therapy, while no significant difference was observed and no deaths related to adverse events occurred.

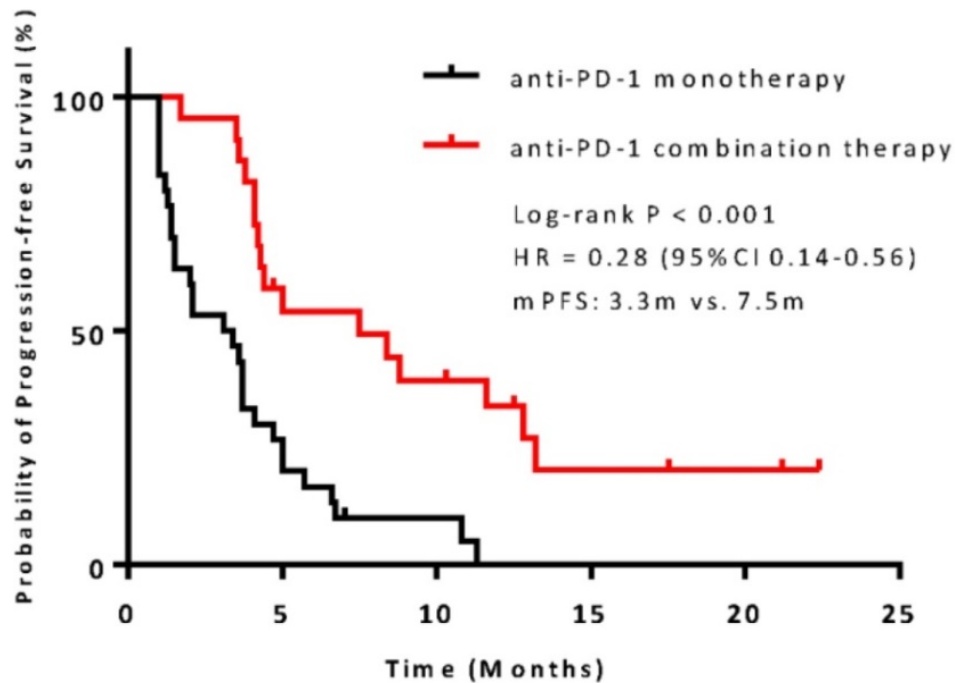

Figure 2. Kaplan-Meier survival curve of progression-free survival comparing anti-PD-1 monotherapy and combination therapy. $\mathrm{Cl}=$ confidence interval; $\mathrm{HR}=$ hazard ratio.

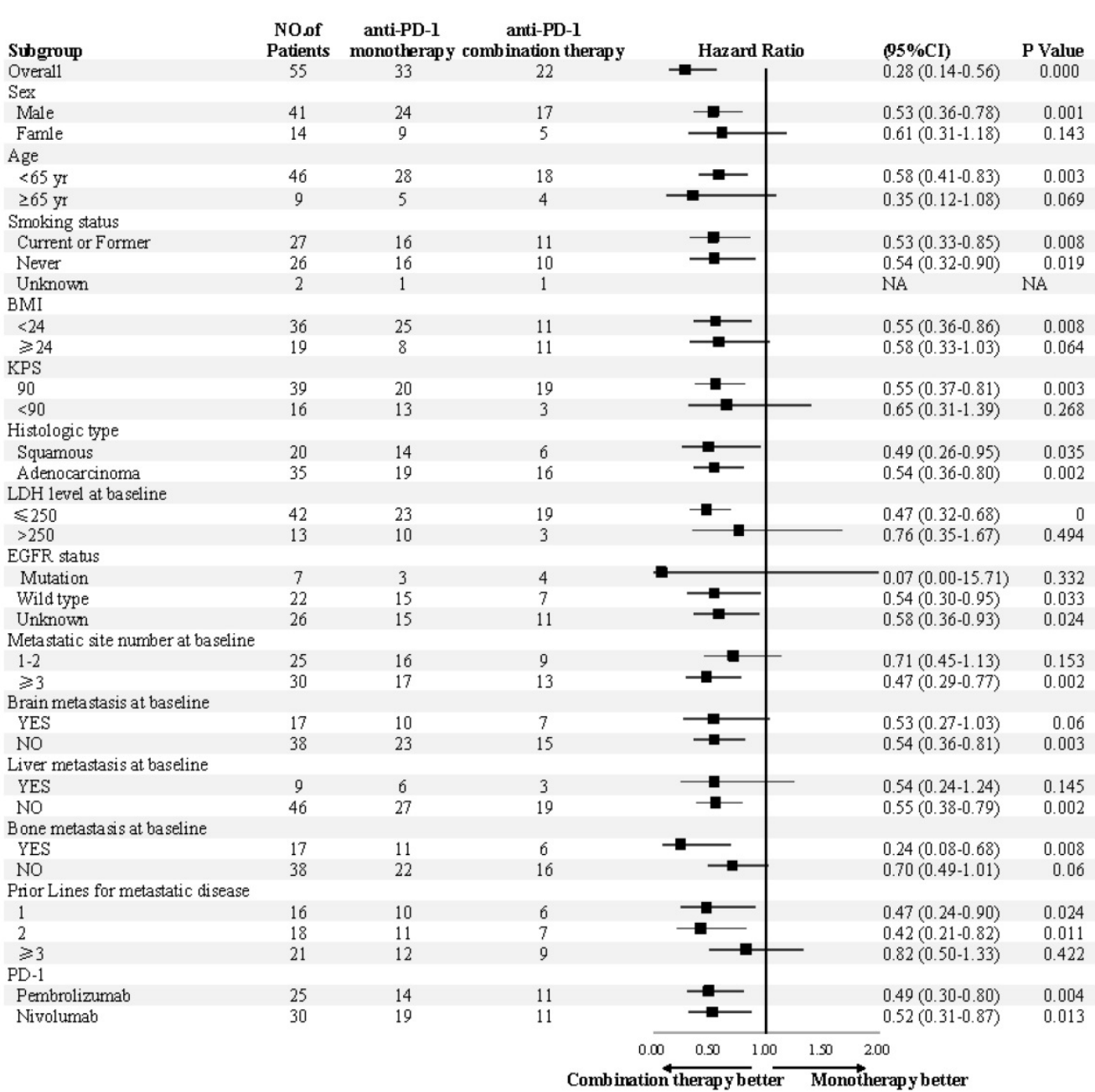

Figure 3. Subgroup analyses of progression-free survival. Subgroup analysis were presented from a Cox proportional-hazards model. 
A

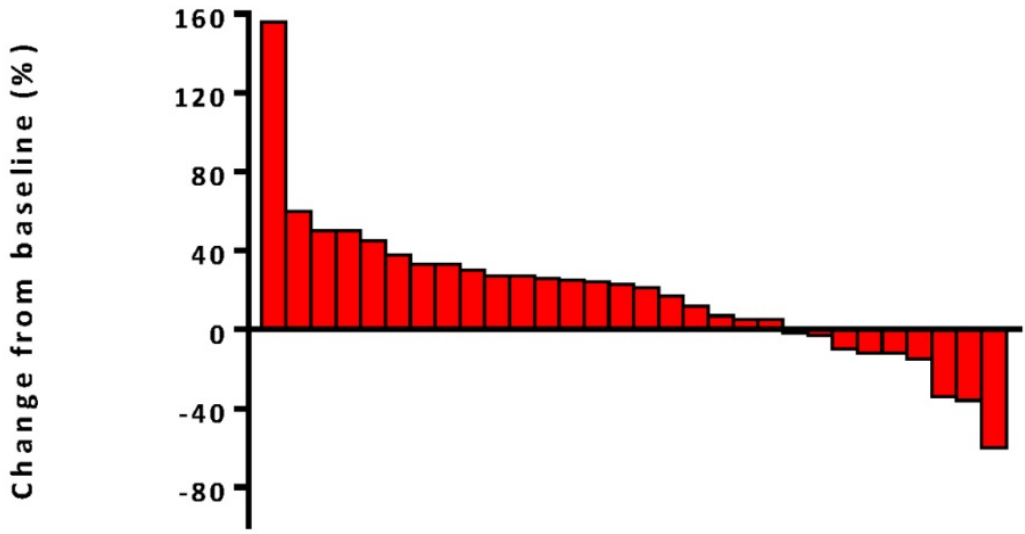

B

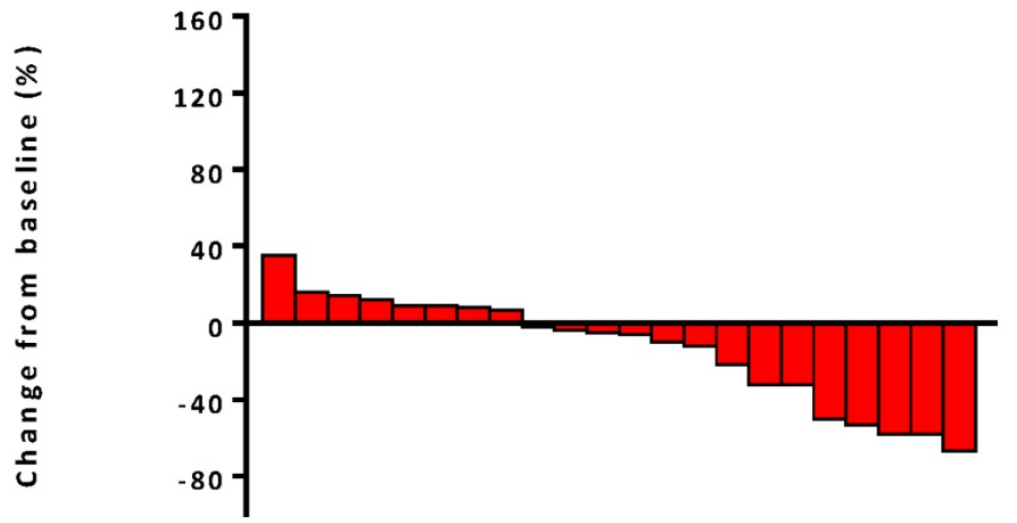

Figure 4. Waterfall plots of best percentage change. (A) The best percentage change from baseline in tumor size for individual patients in anti-PD-1 monotherapy group. (B) The best percentage change from baseline in tumor size for individual patients in anti-PD-1 combination therapy group.

Table 3. Adverse Events.

\begin{tabular}{|c|c|c|c|c|c|c|c|c|}
\hline & \multicolumn{4}{|c|}{ Anti-PD-1 monotherapy $(\mathrm{N}=33)$} & \multicolumn{4}{|c|}{ Anti-PD-1 combination therapy $(\mathrm{N}=22)$} \\
\hline & Grade 1-2 & Grade 3 & Grade 4 & Grade 5 & Grade 1-2 & Grade 3 & Grade 4 & Grade 5 \\
\hline \multicolumn{9}{|l|}{ Treatment related } \\
\hline Any & $27(81.8 \%)$ & $2(6.1 \%)$ & 0 & 0 & $16(72.7 \%)$ & $2(9.1 \%)$ & $3(13.6 \%)$ & 0 \\
\hline Nausea & $4(12.1 \%)$ & $\begin{array}{l}1(3.0 \%)(3.0 \%) \\
(3.0 \%)(3.0 \%)\end{array}$ & 0 & 0 & $6(27.3 \%)$ & 0 & 0 & 0 \\
\hline Fatigue & $8(24.2 \%)$ & 0 & 0 & 0 & $6(27.3 \%)$ & $1(4.5 \%)$ & 0 & 0 \\
\hline Rash & $2(6.1 \%)$ & $1(3.0 \%)(3.0 \%)$ & 0 & 0 & $4(18.2 \%)$ & 0 & 0 & 0 \\
\hline Vomiting & 0 & 0 & 0 & 0 & $3(13.6 \%)$ & 0 & 0 & 0 \\
\hline Leukopenia & $4(12.1 \%)$ & 0 & 0 & 0 & $1(4.5 \%)$ & 0 & $2(9.1 \%)$ & 0 \\
\hline Neutropenia & $1(3.0 \%)$ & 0 & 0 & 0 & $2(9.1 \%)$ & 0 & 0 & 0 \\
\hline Hypothyroidism & 0 & 0 & 0 & 0 & $1(4.5 \%)$ & 0 & 0 & 0 \\
\hline $\begin{array}{l}\text { Increased alanine } \\
\text { aminotransferase }\end{array}$ & 0 & 0 & 0 & 0 & $1(4.5 \%)$ & 0 & 0 & 0 \\
\hline Pneumonitis & $2(6.1 \%)$ & 0 & 0 & 0 & 0 & $2(9.1 \%)$ & $1(4.5 \%)$ & 0 \\
\hline Fever & $5(15.2)$ & $1(3.0 \%)$ & 0 & 0 & 0 & $1(4.5 \%)$ & 0 & 0 \\
\hline Constipation & $1(3.0 \%)$ & 0 & 0 & 0 & 0 & 0 & 0 & 0 \\
\hline Myalgia & $2(6.1 \%)$ & 0 & 0 & 0 & $1(4.5 \%)$ & 0 & 0 & 0 \\
\hline Anemia & $4(12.1 \%)$ & 0 & 0 & 0 & $2(9.1 \%)$ & 0 & 0 & 0 \\
\hline Appetite decreases & $2(6.1 \%)$ & 0 & 0 & 0 & 0 & 0 & 0 & 0 \\
\hline Thrombocytopenia & $1(3.0 \%)$ & 0 & 0 & 0 & $1(4.5 \%)$ & 0 & 0 & 0 \\
\hline
\end{tabular}

In previous studies, the ICIs combination strategies in first-line advanced NSCLC were anti-PD-1 with standard platinum-based chemotherapy in KEYNOTE-021, KEYNOTE-189, KEYNOTE-407, CHECKMATE-012 and CHECKMATE-227 trial, and anti-PD-L1 with standard platinum-based chemotherapy plus bevacizumab in IMpower150 and IMpower 131.19-22,24,27-29 These data has greatly encouraged researchers to combine ICIs with other regimens to maximize the efficacy of immunotherapy. There was no reports of which regimen would be an ideal 
partner with ICIs for NSCLC patients with prior treatment of platinum-based chemotherapy. Nab-paclitaxel is so far the standard chemotherapy for various solid tumors including advanced NSCLC and breast cancer. ${ }^{30,31}$ Preclinical data revealed that taxanes could modulate tumor cell immunogenicity by up-regulating the expression of MHC class I molecules. ${ }^{32}$ Phase IB trial also demonstrated a best ORR of $71 \%$ in triple-negative breast cancer patients treated with atezolizumab combined with nab-paclitaxel which indicated nab-paclitaxel plus ICIs might be a rational combination strategy. ${ }^{33}$ In our study, $90.9 \%$ of the patients in this study have failed after platinum-based chemotherapy previously, and $40.9 \%$ of the patients received nab-paclitaxel plus anti-PD-1. Our data suggested that nab-paclitaxel might be a good addition to improve the sensitivity to anti-PD-1, which needs further validation in the future trials.

Previous studies have demonstrated that ICIs combined with angiogenesis inhibitors was effective. ${ }^{34-37}$ Data from the phase I trial have shown that anti-CTLA-4 ipilimumab combined with bevacizumab was effective in metastatic melanoma, with an ORR of $19.6 \%{ }^{34}$ Other clinical trials also displayed favorable ORR of ICIs plus angiogenesis inhibitors in renal and urothelial carcinoma patients. $35,38,39$ Considering the low ORR of ICIs monotherapy, bevacizumab might be a relatively safe combining choice. In our study, the ORR was $0 \%$ in anti-PD-1 and bevacizumab combination subgroup, although with a DCR of $87.5 \%$. The population who received anti-PD-1 plus bevacizumab in this analysis were heavily treated and under intrinsically refractory conditions. The disappointing efficacy of anti-PD-1 plus bevacizumab may also pull down the total survival benefit in the combination therapy group. Considering that the benefit of anti-PD-1 combination therapy may have been largely driven by the patients treated with chemotherapy-containing combination therapy, the analysis was re-conducted with the exclusion of patients receiving bevacizumab plus anti-PD-1, showing a similar PFS in the combination group (8.0 months) with the previous results (7.5 months). The efficacy of ICIs when combined with bevacizumab in NSCLC patients is needed to be further investigated.

After progression upon first-line treatment, NSCLC patients harboring mutations of EGFR or ALK may be less likely to achieve response to PD-1 inhibitor monotherapy. ${ }^{12,13}$ In KEYNOTE-021, patients harboring EGFR or ALK mutations were excluded. ${ }^{19}$ Results from the IMpower 150 trial revealed that advanced NSCLC patients harboring EGFR or ALK genetic aberrations could also benefit from atezolizumab plus carboplatin/paclitaxel/ bevacizumab therapy compared to carboplatin/paclitaxel/ bevacizumab therapy without atezolizumab. ${ }^{29}$ Results from the BIRCH trial which examined the efficacy of atezolizumab for NSCLC patients have shown that the ORR was $23 \%$ in EGFR mutant patients who haven't received chemotherapy in the advanced setting. ${ }^{40}$ In the present study, patients with driver gene mutations were included. There are three patients with EGFR mutation in the monotherapy group, among whom stable disease was observed in only one patient and progression disease was observed in the other two patients. In the combination group, there are four patients carrying EGFR mutations and one patient carrying $A L K$ mutation. Partial response was observed in 1 of these patients (EGFR mutation) and the other 4 patients were evaluated as stable disease. All these eight patients with driver mutations have been previously treated with chemotherapy and have progressed after at least three prior lines of therapies. It is presumable that combination strategies may provide promising opportunity for these patients with driver mutations.

Patients with advanced disease status who have been heavily treated tend to exhibit a lower KPS status. It is possible that patients with a lower KPS score could not tolerate the toxicity of chemotherapy. In the present study, a slightly higher proportion of patients with good KPS score was observed in the combination group. Although no significant impact on prognosis in terms of KPS score was shown in the multivariable analysis, patients with a good KPS may tend to receive combination therapy in clinical practice. In the combination group, two patients received platinum-doublet chemotherapy plus anti-PD-1. Partial response was observed in one patient with 4-month PFS and stable disease was observed in the other patient with 13-month PFS. The KPS scores of these two patients were both 90 . Considering that patients with platinum-doubletcontaining therapy may result in a favorable prognosis, we re-conducted the analysis with these two patients excluded, which showed a consistently superior PFS in combination group compared with monotherapy group (7.5 months vs. 3.3 months, $\mathrm{P}=$ 0.0003).

Most of the AEs were manageable in this study, although the incidence of grade 3-4 AEs was higher in patients with the combination treatment, which was consistent to the previously reports. ${ }^{4,8,19,22}$ Most of the AEs were mild (grade 1 or 2 ) and well-tolerated. No death occurred due to adverse events.

Our study has several limitations. Despite we prospectively designed the study before the launch; 
the retrospective nature of this study may limit the interpretation of the results. The small sample size also could contribute to the unavoidable selection bias, recall bias and measurement bias, relatively weakening the reliability and validity of our conclusions.

In conclusion, we observed prolonged PFS and elevated DCR in aNSCLC when treated with PD-1 inhibitor plus chemo and/or beva compared with PD-1 inhibitor monotherapy in second-line therapy or beyond setting in this retrospective analysis. Our findings may provide insights into the strategies for managing refractory NSCLC patients and valuable clues for the further prospective study in the future.

\section{Abbreviations}

aNSCLC: advanced NSCLC; ICIs: immune checkpoints inhibitors; PD-1 inhibitor: monoclonal antibodies against programmed cell death protein-1; OS: overall survival; ORR: objective response rate; FDA: Food and Drug Administration; HR: hazard ratio; GHPLA: General Hospital of the People's Liberation Army; AEs: adverse events; TREND: Transparent Reporting of Evaluations with Nonrandomized Designs; DCR: disease control rate.

\section{Supplementary Material}

Supplementary tables.

http://www.jcancer.org/v11p0741s1.pdf

\section{Acknowledgement}

This work was supported by National Natural Science Foundation of China [Grants No. 81402552 to Fan Zhang, 81672996 to $\mathrm{Yi} \mathrm{Hu}$, and 81770204 to Lei Zhao], The Young Talent Program of PLA General Hospital and the Young Talent Foundation of PLA General Hospital (2018XXFC-3 to Fan Zhang, 2018XXFC-11 to Lei Zhao), and National Key R\&D Program of China, Stem Cell and Translation Research (2017YFA0106200 to Fang Yuan).

\section{Competing Interests}

Drs. Yuzi Zhang, Guoqiang Wang, Zhengyi Zhao, and Shangli Cai are employees of 3D Medicines Inc. The remaining authors declare no conflict of interests.

\section{References}

1. Siegel RL, Miller KD, Jemal A. Cancer Statistics, 2017. CA Cancer J Clin. 2017; 67(1): 7-30.

2. Inamura K. Lung Cancer: Understanding Its Molecular Pathology and the 2015 WHO Classification. Front Oncol. 2017; 7: 193

3. Reck M, Rodriguez-Abreu D, Robinson AG, et al. Pembrolizumab versus Chemotherapy for PD-L1-Positive Non-Small-Cell Lung Cancer. N Engl J Med. 2016; 375(19): 1823-1833.

4. Brahmer J, Reckamp KL, Baas P, et al. Nivolumab versus Docetaxel in Advanced Squamous-Cell Non-Small-Cell Lung Cancer. N Engl J Med. 2015; 373(2): 123-135.
5. Borghaei $\mathrm{H}$, Paz-Ares $\mathrm{L}$, Horn $\mathrm{L}$, et al Nivolumab versus Docetaxel in Advanced Nonsquamous Non-Small-Cell Lung Cancer. N Engl J Med. 2015; 373(17): 1627-1639.

6. Herbst RS, Baas P, Kim D-W, et al. Pembrolizumab versus docetaxel for previously treated, PD-L1-positive, advanced non-small-cell lung cancer (KEYNOTE-010): a randomised controlled trial. Lancet. 2016; 387(10027): 1540-1550.

7. Julie B, Leora H, David J, et al. Abstract CT077: Five-year follow-up from the CA209-003 study of nivolumab in previously treated advanced non-small cell lung cancer (NSCLC): Clinical characteristics of long-term survivors. AACR Annual Meeting 2017. 2017; 77(13).

8. Garon EB, Rizvi NA, Hui R, et al. Pembrolizumab for the treatment of non-small-cell lung cancer. N Engl J Med. 2015; 372(21): 2018-2028.

9. Rittmeyer A, Barlesi F, Waterkamp D et al. Atezolizumab versus docetaxel in patients with previously treated non-small-cell lung cancer (OAK): a phase 3, open-label, multicentre randomised controlled trial. Lancet. 2017; 389(10066): 255-265.

10. Carbone DP, Reck M, Paz-Ares L, et al. First-Line Nivolumab in Stage IV or Recurrent Non-Small-Cell Lung Cancer. N Engl J Med. 2017; 376(25): 2415-2426.

11. Lemery S, Keegan P, Pazdur R. First FDA approval agnostic of cancer siteWhen a biomarker defines the indication. N Engl J Med. 2017; 377(15): 1409-1412.

12. Gainor JF, Shaw AT, Sequist LV, et al. EGFR Mutations and ALK Rearrangements Are Associated with Low Response Rates to PD-1 Pathway Blockade in Non-Small Cell Lung Cancer: A Retrospective Analysis. Clin Cancer Res. 2016; 22(18): 4585-4593.

13. Dong ZY, Zhang JT, Liu SY, et al. EGFR mutation correlates with uninflamed phenotype and weak immunogenicity, causing impaired response to PD-1 blockade in non-small cell lung cancer. Oncoimmunology. 2017; 6(11): e1356145.

14. Lee CK, Man J, Lord S, et al. Checkpoint Inhibitors in Metastatic EGFR-Mutated Non-Small Cell Lung Cancer-A Meta-Analysis. J Thorac Oncol. 2017; 12(2): 403-407.

15. Hu-Lieskovan S RA. New Combination Strategies Using Programmed Cell Death 1/Programmed Cell Death Ligand 1 Checkpoint Inhibitors as a Backbone. Cancer J. 2017; 23(1): 10-22.

16. Liu WM, Fowler DW, Smith $\mathrm{P}$, et al. Pre-treatment with chemotherapy can enhance the antigenicity and immunogenicity of tumours by promoting adaptive immune responses. Br J Cancer. 2010; 102(1): 115-123.

17. Pircher A, Wolf D, Heidenreich A, et al. Synergies of Targeting Tumor Angiogenesis and Immune Checkpoints in Non-Small Cell Lung Cancer and Renal Cell Cancer: From Basic Concepts to Clinical Reality. Int J Mol Sci. 2017; 18(11): E2291.

18. Tabchi S, Blais N. Antiangiogenesis for Advanced Non-Small-Cell Lung Cancer in the Era of Immunotherapy and Personalized Medicine. Front Oncol. 2017; 7:52.

19. Langer CJ, Gadgeel SM, Borghaei $\mathrm{H}$, et al. Carboplatin and pemetrexed with or without pembrolizumab for advanced, non-squamous non-small-cell lung cancer: a randomised, phase 2 cohort of the open-label KEYNOTE-021 study. Lancet Oncol. 2016; 17(11): 1497-1508

20. Gandhi L, Rodriguez-Abreu D, Gadgeel S, et al. Pembrolizumab plus Chemotherapy in Metastatic Non-Small-Cell Lung Cancer. N Engl J Med. 2018; 378(22): 2078-2092.

21. Paz-Ares L, Luff A, Vicente D, et al. Pembrolizumab plus Chemotherapy for Squamous Non-Small-Cell Lung Cancer. N Engl J Med. 2018; 379(21): 2040-2051.

22. Rizvi NA, Hellmann MD, Brahmer JR, et al. Nivolumab in Combination With Platinum-Based Doublet Chemotherapy for First-Line Treatment of Advanced Non-Small-Cell Lung Cancer. J Clin Oncol. 2016; 34(25): 2969-2979.

23. M Reck, Socinski MA, Cappuzzo F, et al. Primary PFS and safety analyses of a randomized phase III study of carboplatin + paclitaxel + / - bevacizumab, with or without atezolizumab in $1 \mathrm{~L}$ non-squamous metastatic nsclc (IMPOWER150). Ann Oncol. 2017; 28 (Suppl 11).

24. Jotte MR, Cappuzzo F, Vynnychenko I, et al. IMpower131: Primary PFS and safety analysis of a randomized phase III study of atezolizumab + carboplatin + paclitaxel or nab-paclitaxel vs carboplatin + nab-paclitaxel as $1 \mathrm{~L}$ therapy in advanced squamous NSCLC. J Clin Oncol. 2018; 36 (Suppl 18): LBA9000.

25. Eisenhauer EA, Therasse $P$, Bogaerts J, et al. New response evaluation criteria in solid tumours: revised RECIST guideline (version 1.1). Eur J Cancer. 2009; 45(2): 228-247.

26. Des Jarlais DC LC, Crepaz N, TREND Group. Improving the reporting quality of nonrandomized evaluations of behavioral and public health interventions: the TREND statement. Am J Public Health. 2004; 94(3): 361-366.

27. Weiss GJ, Waypa J, Blaydorn L, et al. A phase Ib study of pembrolizumab plus chemotherapy in patients with advanced cancer (PembroPlus). Br J Cancer. 2017; 117(1): 33-40

28. Hellmann MD, Ciuleanu TE, Pluzanski A, et al. Nivolumab plus Ipilimumab in Lung Cancer with a High Tumor Mutational Burden. N Engl J Med. 2018; 378(22): 2093-2104

29. Reck M, Mok TSK, Nishio M, et al. Atezolizumab plus bevacizumab and chemotherapy in non-small-cell lung cancer (IMpower150): key subgroup analyses of patients with EGFR mutations or baseline liver metastases in a randomised, open-label phase 3 trial. Lancet Respir Med. 2019; 7(5): 387-401. 
30. Gradishar WJ, Tjulandin S, Davidson N, et al. Phase III trial of nanoparticle albumin-bound paclitaxel compared with polyethylated castor oil-based paclitaxel in women with breast cancer. J Clin Oncol. 2005; 23(31): 7794-7803.

31. Socinski MA, Bondarenko I, Karaseva NA, et al. Weekly nab-paclitaxel in combination with carboplatin versus solvent-based paclitaxel plus carboplatin as first-line therapy in patients with advanced non-small-cell lung cancer: final results of a phase III trial. J Clin Oncol. 2012; 30(17): 2055-2062.

32. Chen G, Emens LA. Chemoimmunotherapy: reengineering tumor immunity. Cancer Immunol Immunother. 2013; 62(2): 203-216.

33. Adams S, Diamond JR, Hamilton EP, et al. Phase $\mathrm{Ib}$ trial of atezolizumab in combination with nab-paclitaxel in patients with metastatic triple-negative breast cancer (mTNBC). J Clin Oncol. 2016; 34 (Suppl 15): 1009-1009.

34. Hodi FS, Lawrence D, Lezcano C, et al. Bevacizumab plus ipilimumab in patients with metastatic melanoma. Cancer Immunol Res. 2014; 2(7): 632-642.

35. Apolo AB, Mortazavi A, Stein MN, et al. A phase I study of cabozantinib plus nivolumab (CaboNivo) and cabonivo plus ipilimumab (CaboNivolpi) in patients (pts) with refractory metastatic (m) urothelial carcinoma (UC) and other genitourinary (GU) tumors. J Clin Oncol. 2017: 35 (Suppl 6): 293-293.

36. Lee $\mathrm{CH}$, Makker V, Rasco D, et al. A phase $1 \mathrm{~b} / 2$ trial of lenvatinib plus pembrolizumab in patients with renal cell carcinoma. Ann Oncol. 2017; 28 (Suppl 5): v295-v329.

37. Makker V, Rasco DW, Dutcus CE, et al. A phase Ib/II trial of lenvatinib (LEN) plus pembrolizumab (Pembro) in patients (Pts) with endometrial carcinoma. J Clin Oncol. 2017; 35 (Suppl 15): 5598-5598.

38. Wallin JJ, Bendell JC, Funke R, et al. Atezolizumab in combination with bevacizumab enhances antigen-specific T-cell migration in metastatic renal cell carcinoma. Nat Commun. 2016; 7: 12624.

39. Atkins MB, McDermott DF, Powles T, et al. IMmotion150: A phase II trial in untreated metastatic renal cell carcinoma (mRCC) patients (pts) of atezolizumab (atezo) and bevacizumab (bev) vs and following atezo or sunitinib (sun). J Clin Oncol. 2017; 35 (Suppl 15): 4505-4505.

40. Peters S, Gettinger S, Johnson ML, et al. Phase II trial of atezolizumab as first-line or subsequent therapy for patients with programmed death-ligand 1-selected advanced Non-Small-Cell Lung Cancer (BIRCH). J Clin Oncol. 2017; 35(24): 2781-2789. 